\section{RSP}

http://www.rsp.fsp.usp.br/
Revista de Saúde Pública

\title{
Variabilidade climática aumenta a morbimortalidade associada ao material particulado
}

Poliany Cristiny de Oliveira Rodrigues', Samya de Lara Pinheiro", Washington Junger"', Eliane Ignotti', Sandra de Souza Hacon ${ }^{\mathrm{IV}}$

1 Universidade do Estado de Mato Grosso. Cáceres, MT, Brasil

" Aria do Brasil. Rio de Janeiro, RJ, Brasil

III Universidade do Estado do Rio de Janeiro. Rio de Janeiro, RJ, Brasil

Iv Escola Nacional de Saúde Pública. Fundação Oswaldo Cruz. Rio de Janeiro, RJ, Brasil

\section{RESUMO}

OBJETIVO: Analisar se o material particulado fino $\left(\mathrm{PM}_{25}\right)$, bem como seu efeito sinérgico com a temperatura máxima, a umidade e as estações do ano estão associados à morbimortalidade por doenças cardiovasculares.

MÉTODOS: Estudo ecológico de séries temporais. Foram utilizados como desfechos os registros diários de óbito e internação em adultos com 45 anos ou mais de idade para os anos de 2009 a 2011 nos municípios de Cuiabá e Várzea Grande, Mato Grosso, Brasil. Utilizou-se regressão de Poisson via modelos aditivos generalizados, assumindo-se um nível de significância de 5\%. O modelo foi controlado para tendência temporal, sazonalidade, temperatura média, umidade e efeitos de calendário. Concentrações diárias de $\mathrm{PM}_{2.5}$ (material particulado com diâmetro aerodinâmico inferior a 2,5 micrômetros) foram obtidas por meio da conversão dos valores da espessura ótica de aerossóis. Temperatura máxima, umidade e estações do ano foram incluídas separadamente ao modelo como variáveis indicadoras para análise do efeito sinérgico do $\mathrm{PM}_{2.5}$ com a morbimortalidade por doenças cardiovasculares. Foi calculado o aumento percentual de risco relativo (\%RR) dos óbitos e internações para o aumento linear de $10 \mu \mathrm{g} / \mathrm{m}^{3} \mathrm{de} \mathrm{PM}_{2,5}$.

Correspondência:

Poliany Cristiny de Oliveira Rodrigues Departamento de Enfermagem -

UNEMAT

Avenida São João, s/n

78200-000 Cáceres, MT, Brasil

E-mail: polianyrodrigues@unemat.br

Recebido: 3 fev 2016

Aprovado: 2 out 2016

Como citar: Rodrigues PCO, Pinheiro SL, Junger W, Ignotti E, Hacon SS. Variabilidade climática aumenta a morbimortalidade associada ao material particulado. Rev Saude Publica. 2017;51:91.

Copyright: Este é um artigo de acesso aberto distribuído sob os termos da Licença de Atribuição Creative Commons, que permite uso irrestrito, distribuição e reprodução em qualquer meio, desde que o autor e a fonte originais sejam creditados.
RESULTADOS: Entre 2009 e 2011, o aumento de PM $_{2,5}$ foi associado a um \%RR 2,28 (IC95\% 0,53-4,06) para internações no mesmo dia de exposição e \%RR 3,57 (IC95\% 0,82-6,38) para óbitos com uma defasagem de três dias. Em dias quentes, observou-se \%RR 4,90 (IC95\% -0,61-9,38) para óbitos. Não foi observada modificação de efeito do $\mathrm{PM}_{25}$ pela temperatura máxima relacionada as internações. Em dias com baixa umidade, o \%RR foi 5,35 (IC95\% -0,20-11,22) para óbitos e 2,71 (IC95\% -0,39-5,92) para internações. Na estação seca, o \%RR foi 2,35 (IC95\% 0,59-4,15) para internações e 3,43 (IC95\% 0,58-6,35) para óbitos.

CONCLUSÕES: $\mathrm{O}_{2 M_{2,5}}$ está associado à morbimortalidade por doenças cardiovasculares e seus efeitos podem ser potencializados pelo calor, pela baixa umidade e durante a estação seca.

DESCRITORES: Material Particulado, efeitos adversos. Poluentes do Ar. Doenças Cardiovasculares, epidemiologia. Fatores de Risco. Estações do Ano. Estudos Ecológicos. Estudos de Séries Temporais. 


\section{INTRODUÇÃO}

Existem evidências de que a exposição ao material particulado fino $\left(\mathrm{PM}_{2.5}\right)$ está associada a um maior risco de mortalidade, internação e exacerbação de doenças cardiovasculares (DC). A magnitude dos efeitos relatados, no entanto, está relacionada à composição, quantidade, porta de entrada, capacidade de transporte e deposição do PM no organismo. O PM ${ }_{2,5}$ apresenta maior toxicidade devido a sua multielementariedade e porque pode atingir tanto as porções mais inferiores do trato respiratório quanto entrar em contato diretamente com a corrente sanguínea ${ }^{5}$.

A localização geográfica e a sazonalidade climática também podem influenciar na magnitude dos efeitos adversos do PM sobre a saúde humana. Alguns estudos têm mostrado que o clima pode modificar a associação do $\mathrm{PM}_{2.5}$ com a morbimortalidade. Apesar de a temperatura ser o parâmetro mais estudado ${ }^{8,10,17,23}$, alguns autores observaram que a umidade, a pressão atmosférica e as estações do ano também podem agir como modificadores do efeito do PM sobre os eventos de saúde ${ }^{14,16}$.

Na Amazônia e no Cerrado, particularmente, os fenômenos climáticos intensificam os efeitos à saúde relacionados à poluição atmosférica. Nessas regiões, alguns estudos ecológicos mostram que o PM está associado ao aumento da morbimortalidade por doenças respiratórias ${ }^{6,22} \mathrm{e}$ cardiovasculares ${ }^{12,19}$, em crianças e idosos, principalmente durante o período de seca, no qual ocorre a queima acidental ou intencional de pastagens, cana-de-açúcar e floresta. Contudo, os efeitos modificadores da sazonalidade climática da região sobre a morbimortalidade associada à poluição atmosférica ainda não foram investigados.

Caracterizar os efeitos do $\mathrm{PM}_{2,5}$, bem como seu efeito conjunto com as variáveis climáticas, pode explicar melhor a heterogeneidade dos efeitos observados na região e subsidiar ações de mitigação e controle mais efetivas e adequadas às necessidades da população local relacionadas à poluição do ar. Esse estudo teve por objetivo analisar se o material particulado fino $\left(\mathrm{PM}_{2,5}\right)$, bem como seu efeito sinérgico com a temperatura máxima, a umidade e as estações do ano estão associados à morbimortalidade por doenças cardiovasculares.

\section{MÉTODOS}

Estudo ecológico de séries temporais dos registros diários de óbitos e internações por doenças cardiovasculares. Foram selecionados indivíduos de 45 anos ou mais de idade residentes dos municípios de Cuiabá e Várzea Grande que foram internados e que foram a óbito por DC (Capítulo IX da Décima Revisão da Classificação Internacional de Doenças [CID-10] códigos I00 a I99) entre 23 de abril de 2009 e 31 de dezembro de 2011. A série temporal de 2009 a 2011, composta por 983 dias, foi selecionada devido à disponibilidade de dados de espessura ótica de aerossóis que possibilitou as estimativas de $\mathrm{PM}_{2,5}$.

a Instituto Brasileiro de

Geografia e Estatística. IBGE Cidades: indicadores e dados populacionais. Rio de Janeiro (DF); IBGE; 2010 [citado 6 mai 2013]. Disponível em: http://www.ibge. gov.br/cidadesat/xtras/perfil.

b Instituto Nacional de Meteorologia. Dados históricos. Brasília (DF): INMET; 2013 [citado 15 jun 2013]. Disponível em: http://www.inmet.gov.br/ portal/php?codmun=510340 \&search=mato-grosso $\mid$ cuiaba cMinistério da Saúde (BR), Departamento de Informática do SUS (DATASUS). Rede Interagencial de Informações para a Saúde - RIPSA. Indicadores e dados básicos - Brasil -2012 IDB 2012. Brasília (DF); 2012 [citado 13 mai 2014]. Disponível em: http://tabnet.datasus.gov.br/ cgi/idb2012/matriz.htm
As cidades de Cuiabá e Várzea Grande, localizadas no Estado de Mato Grosso, Brasil, formam uma conurbação que agrupa uma população de aproximadamente 820 mil habitantes, cerca de $90 \%$ da população total da região metropolitana do Vale do Rio Cuiabá. A população com 45 anos ou mais de idade representa aproximadamente $60 \%$ da população total das cidades ${ }^{a}$.

O clima da região é limitado em duas estações marcantes: a seca e a chuvosa. Entre os meses de maio a outubro, período de seca, a prevalência de queimadas é historicamente elevada na região. O período mais intenso de chuvas corresponde aos meses de novembro a abril. As cidades são muito conhecidas por seu forte calor de, em média, $32^{\circ} \mathrm{C}$ diários. A temperatura máxima média chega aos $41^{\circ} \mathrm{C}$ nos meses mais quentes ${ }^{b}$. As DC aparecem como a primeira causa de mortalidade geral entre 2009 e 2011, com taxa de mortalidade média de 135,35 óbitos a cada 100 mil habitantes, seguindo o perfil de mortalidade do Estado ${ }^{c}$. No mesmo período, as afecções do aparelho circulatório foram a quinta causa de internação no Estado de Mato Grosso e a terceira causa de internação em Cuiabá e Várzea Grande, com uma taxa de internação média de 46,25 internações por 10 mil habitantesc . 
Os registros de mortalidade e internação por DC utilizados como desfechos foram obtidos, respectivamente, da base de dados do Sistema de Informação para a Mortalidade (SIM/SUS) e Sistema de Informações Hospitalares (SIH/SUS). Informações das médias diárias de temperatura e umidade foram provenientes do Instituto Nacional de Meteorologia (INMET).

Dados de Aerosol Optical Depth (AOD) foram obtidos da estação Cuiabá-Miranda (Latitude:-15,43; Longitude: -56,01) disponíveis no site do Aerosol Robotic Network (AERONET). Estimativas de $\mathrm{PM}_{2,5}$ foram geradas por meio da conversão dos valores $\mathrm{AOD}$ (500 nanômetros), por meio de um método desenvolvido e validado para a Amazônia Brasileira e Cerrado ${ }^{\mathrm{d}}$. A base de cálculo desse método permite a obtenção dos valores das concentrações de $\mathrm{PM}_{2.5}$ muito próximos aos dados medidos por estações de monitoramento do $\mathrm{ar}^{2}$ e tem sido utilizada para suprir a carência de informações de poluição atmosférica em algumas áreas ${ }^{1,19}$. Apesar de as medidas obtidas representarem valores médios para toda a coluna atmosférica, com uma resolução espacial de até $10 \mathrm{~km}$, a validação ${ }^{\mathrm{d}}$ mostrou que a conversão é adequada para $\mathrm{o}$ arco do desmatamento e $\mathrm{o} \mathrm{PM}_{2,5}$ estimado obtido pode ser utilizado como alternativa para estudos de impacto do PM na saúde humana².

Foi realizada uma regressão de séries temporais, construindo-se modelos explicativos para contagens de internações e óbitos por DC ao longo do tempo. O método de séries temporais tem sido amplamente aplicado em estudos epidemiológicos ecológicos, principalmente na avaliação do efeito agudo, porque apresenta melhor desempenho na análise dos efeitos lineares da poluição do ar sobre a mortalidade por $\mathrm{DC}^{10,13}$.

Utilizou-se a classe de modelos aditivos generalizados (GAM), com a opção de regressão de Poisson, assumindo-se um nível de significância de $5 \%$. A tendência temporal e a sazonalidade da série foram controladas introduzindo-se as seguintes variáveis: dias da semana e um natural cubic spline do tempo com nove graus de liberdade. Para as variáveis meteorológicas, utilizou-se natural cubic spline da temperatura média e da umidade relativa do ar, com três e dois graus de liberdade, respectivamente. No processo de modelagem, buscou-se reduzir o critério de informação de Akaike (AIC) e melhorar a autocorrelação parcial (PACF).

Optou-se pela utilização do modelo GAM porque não é necessário definir a priori as relações e estruturas entre o indicador de saúde e as variáveis explicativas. Além disso, utilizou-se a biblioteca ARES2 disponível no aplicativo R como uma ferramenta para definir essas relações. O modelo de regressão de Poisson, com função link logarítmica, foi escolhido porque dados de mortalidade obtidos por meio de contagem possuem distribuição de probabilidades do tipo Poisson ${ }^{7,13}$.

Foi calculado o aumento percentual de risco relativo (\%RR) dos óbitos e das internações por DC. Investigou-se as associações da exposição no dia corrente com defasagens simples (single Lag) e o efeito cumulativo de até 10 dias utilizando um modelo polinomial de defasagens distribuídas (distributed Lag). O período de latência de 10 dias foi selecionado para permitir uma estimativa mais precisa dos efeitos do $\mathrm{PM}_{2,5}$ na mortalidade e nas internações por DC, uma vez que esses efeitos podem não ocorrer de forma imediata ou no mesmo dia da exposição $0^{5,18,23}$. O \%RR corresponde ao aumento linear de $10 \mu \mathrm{g} / \mathrm{m}^{3}$ nos níveis de $\mathrm{PM}_{2,5}$.

O efeito sinérgico entre $\mathrm{PM}_{2,5}$ e temperatura máxima, umidade e estação do ano foi testado por meio da estratificação dos modelos finais contruídos para as internações e para os óbitos. O método da estratificação consiste na inserção de uma variável indicadora nos modelos finais construídos, observando-se as novas características do mesmo modelo com a estratificação ${ }^{7}$. Optou-se pela estratificação por esse método utilizar menos parâmetros e oferecer uma comparação simples e quantitativa dos efeitos estimados dos poluentes nos diferentes estratos, em comparação com outros métodos de detecção do efeito modificador ${ }^{10,13}$. Foram incluídas, separadamente, três variáveis indicadoras (dummy) ao modelo: (i) percentil 90 da temperatura máxima $\left(37,9^{\circ} \mathrm{C}\right)$; (ii) percentil 10 da umidade relativa do ar (54,5\%) e (iii) período de seca (maio - outubro) e chuva (janeiro a março, novembro e dezembro). Testou-se a significância estatística das diferenças entre as estimativas do efeito entre os estratos estabelecidos pelas variáveis indicadoras pelo cálculo do intervalo de confiança de 95\% (IC95\%) e do p-valor? 
As análises foram realizadas no aplicativo R 3.0.2 por meio da biblioteca Ares2 ${ }^{7}$. Este estudo foi aprovado pelo Comitê de Ética da Escola Nacional de Saúde Pública (CAAE 18634613.0.0000.5240).

\section{RESULTADOS}

Durante todo o período de estudo foram registradas 8.610 internações e 3.024 óbitos. O material particulado e a umidade relativa do ar mostraram distribuição heterogênea durante o ano, apresentando desvios padrão (DP) de $15,66 \mu \mathrm{g} / \mathrm{m}^{3}$ e $11,35 \%$, respectivamente. Os valores máximos das temperaturas média e máxima são quase três vezes mais elevados que seus respectivos valores mínimos (Tabela 1).

A temperatura média e a temperatura máxima apresentaram correlação direta de cerca de 0,25 com o $\mathrm{PM}_{2,5}$. A umidade apresentou correlação inversa com todas as variáveis, apresentando-se fortemente associada com a temperatura máxima (-0.600). Os óbitos e as internações por DC apresentaram correlação linear abaixo de 0,100 com as variáveis meteorológicas e de poluição (Tabela 2).

Na modelagem pelo método de defasagem simples, observamos um efeito cíclico de redução e aumento a cada três dias, tanto para as internações quanto para os óbitos associados ao aumento linear de $\mathrm{PM}_{2,5}$. Contudo, o efeito apresentou-se mais agudo para as internações. As internações por DC apresentaram \%RR 2,28 (IC95\% 0,53-4,06) para o mesmo dia de exposição (Lag0) e \%RR 2,00 (IC95\% 0,31-3,71) para a defasagem simples de um dia (Lag1). A mortalidade apresentou \%RR 3,57 (IC95\% 0,82-6,38) para a defasagem simples de três dias (Lag3) (Figura 1).

O \%RR acumulado para o período de 10 dias foi 1,81 (IC95\% 0,03-3,61) para óbitos e 2,64 (IC95\% 1,60-3,69) para as internações relacionados ao aumento do $\mathrm{PM}_{2.5}$. O método de defasagens distribuídas reforçou o padrão agudo do efeito para as internações. Observou-se aumento no \%RR da mortalidade a partir do segundo dia de exposição e diminuição do \%RR para as internações ao longo dos dias (Figura 2).

Tabela 1. Estatísticas descritivas das variáveis ambientais e de saúde em estudo. Cuiabá eVárzea Grande, MT, Brasil, 2009 a 2011. $(n=983)$

\begin{tabular}{lccccc}
\hline Variável & Dias sem informação & Média & Desvio padrão & Mínimo & Máximo \\
\hline Óbito por DC $(\mathrm{n})$ & 0 & 3,08 & 1,74 & 0,00 & 9,00 \\
Internação por DC $(\mathrm{n})$ & 0 & 8,76 & 3,55 & 1,00 & 20,00 \\
$\mathrm{PM}_{2,5}\left(\mu \mathrm{g} / \mathrm{m}^{3}\right)$ & $55^{*}$ & 17,07 & 15,66 & 0,10 & 172,30 \\
Temperatura média $\left({ }^{\circ} \mathrm{C}\right)$ & 0 & 26,45 & 3,04 & 11,44 & 33,44 \\
Temperatura máxima $\left({ }^{\circ} \mathrm{C}\right)$ & 0 & 33,69 & 3,88 & 13,30 & 42,30 \\
Umidade relativa do ar $(\%)$ & 0 & 70,71 & 11,35 & 35,00 & 97,00 \\
\hline
\end{tabular}

DC: doenças cardiovasculares; $\mathrm{PM}_{2,5}$ : material particulado fino

* Espaços vazios são comuns em séries provenientes de sensoriamento remoto devido a dias nublados, nuvens e fumaça. Corresponderam a apenas $0,17 \%$ da série e em dias não agrupados.

Tabela 2. Matriz de correlação de Pearson para as variáveis ambientais e de saúde em estudo. Cuiabá e Várzea Grande, MT, Brasil, 2009 a 2011.

\begin{tabular}{lcccccc}
\hline Variável & $\begin{array}{c}\text { Óbitos por } \\
\text { DC }\end{array}$ & $\begin{array}{c}\text { Internações } \\
\text { por DC }\end{array}$ & $\mathbf{P M}_{2,5}$ & $\begin{array}{c}\text { Temperatura } \\
\text { média }\end{array}$ & $\begin{array}{c}\text { Temperatura } \\
\text { máxima }\end{array}$ & Umidade \\
\hline Óbitos por DC & 1 & & & & & \\
Internações por DC & $0,069^{*}$ & 1 & & & & \\
PM $_{2,5}$ & 0,011 & 0,009 & 1 & & \\
Temperatura média & $-0,097^{*}$ & $-0,057$ & $0,262^{*}$ & 1 & & \\
Temperatura máxima & $-0,066$ & $-0,009$ & $0,250^{*}$ & $0,850^{*}$ & 1 & \\
Umidade & $-0,024$ & $-0,039$ & $-0,189^{*}$ & $-0,383^{*}$ & $-0,600^{*}$ & 1 \\
\hline
\end{tabular}

DC: doenças cardiovasculares; $\mathrm{PM}_{2,5}$ : material particulado fino

$* p<0,05$ 
Na estação seca, cerca de $50 \%$ das observações da temperatura máxima estiveram entre $34,8^{\circ} \mathrm{C}$ e $39,10^{\circ} \mathrm{C}$, enquanto aproximadamente $50 \%$ dos dados de umidade relativa do ar estiveram entre $66 \%$ e $51 \%$. A estação chuvosa apresentou cerca de $50 \%$ das observações entre $33^{\circ} \mathrm{C}$ e $36,4^{\circ} \mathrm{C}$, enquanto aproximadamente $50 \%$ dos dados de umidade relativa do ar estiveram entre $77 \%$ e $65 \%$. A média de $\mathrm{PM}_{2,5}$ foi de $18,71 \mu \mathrm{g} / \mathrm{m}^{3}\left(\mathrm{DP}=19,59 \mu \mathrm{g} / \mathrm{m}^{3}\right)$ e $14,88 \mu \mathrm{g} / \mathrm{m}^{3}$ $\left(\mathrm{DP}=7,18 \mu \mathrm{g} / \mathrm{m}^{3}\right)$ nas estações seca e chuvosa, respectivamente.
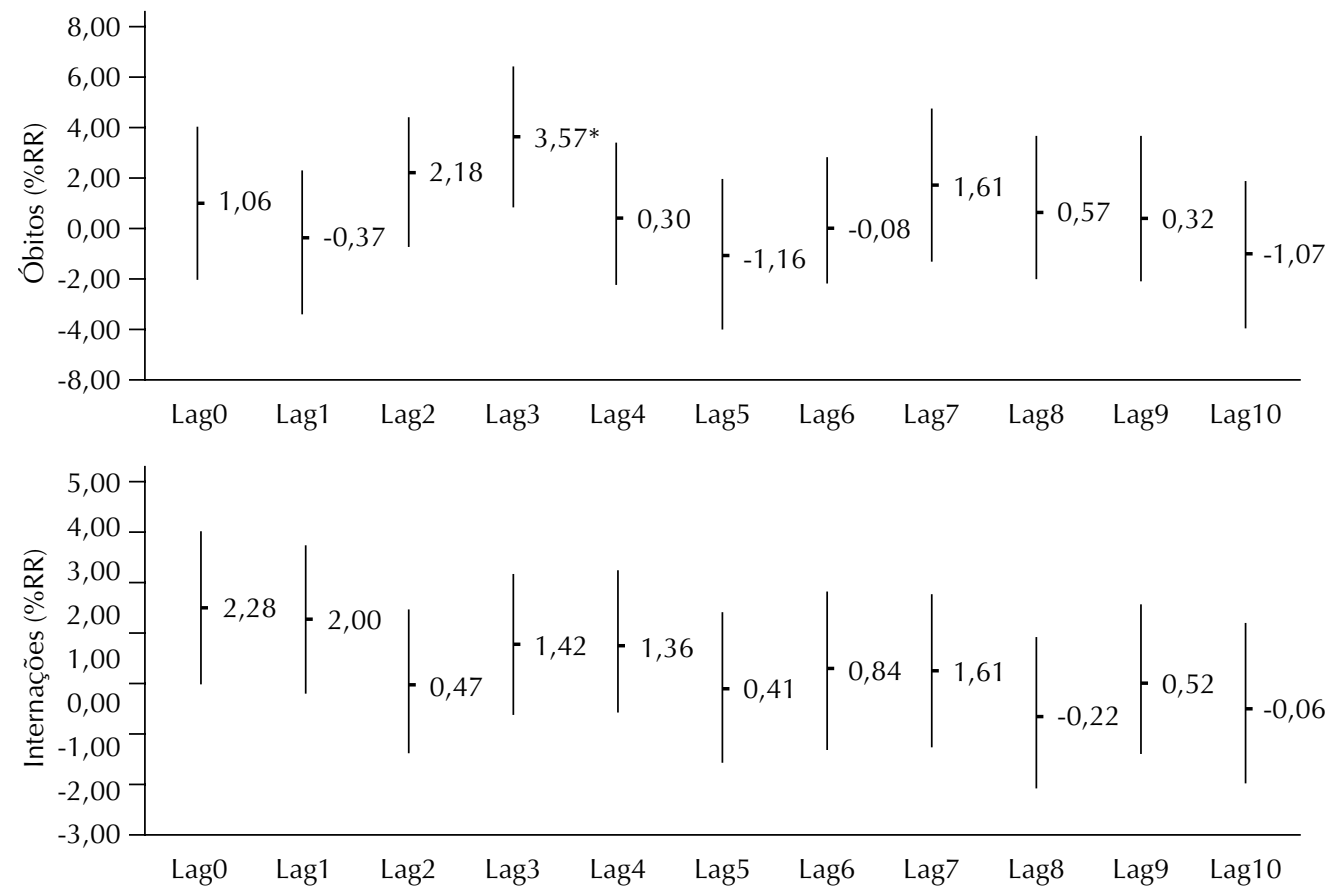

$* \mathrm{p} \leq 0,05$

Figura 1. Risco relativo percentual (\%RR) para internação e mortalidade por doenças cardiovasculares relacionadas a incrementos de $10 \mu \mathrm{g} / \mathrm{m}^{3}$ de $\mathrm{PM}_{2,5}$ por defasagem simples. Cuiabá e Várzea Grande, MT, Brasil, 2009 a 2011.
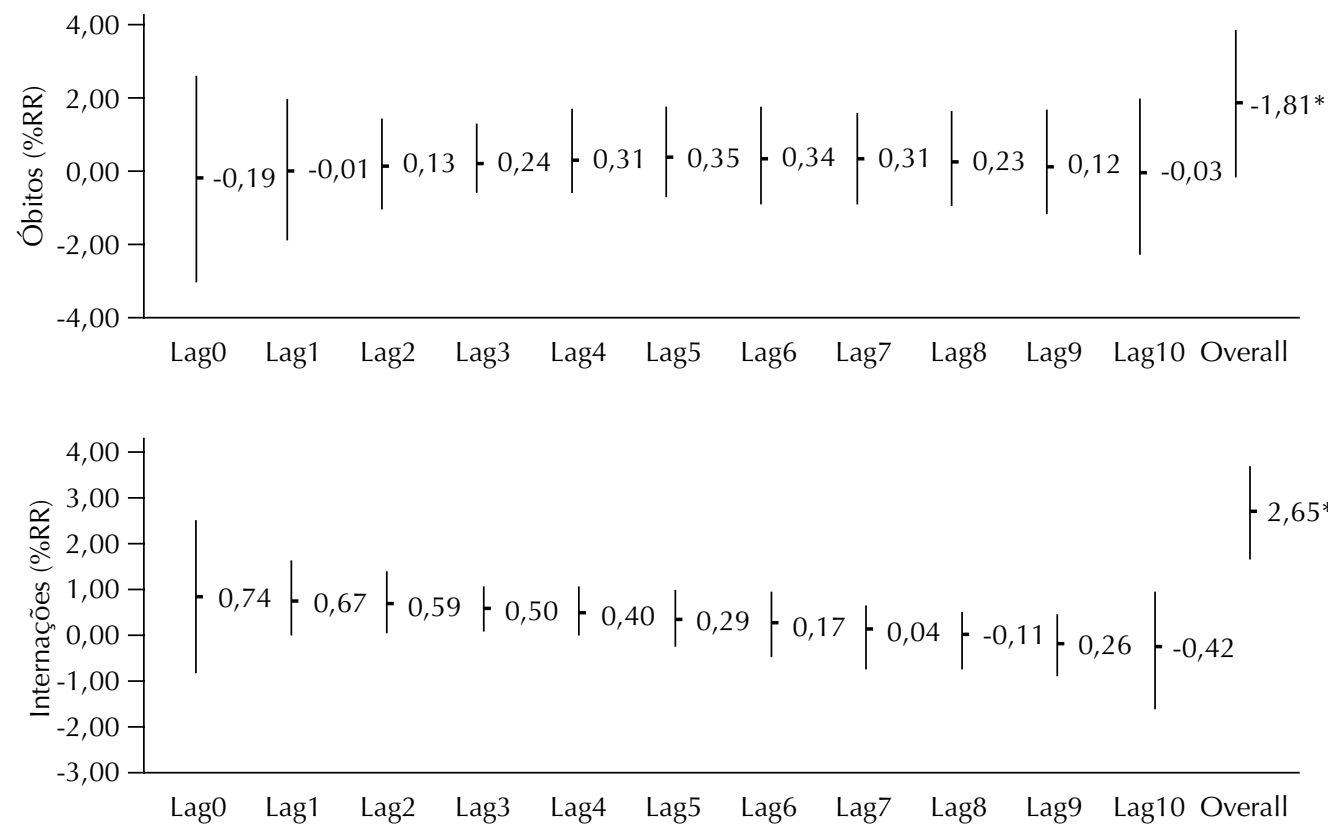

$* p \leq 0,05$

Figura 2. Risco relativo percentual (\%RR) para mortalidade e internação por doenças cardiovasculares relacionadas a incrementos de $10 \mu \mathrm{g} / \mathrm{m}^{3}$ de $\mathrm{PM}_{2,5}$ por distribuição polinomial. Cuiabá e Várzea Grande, MT, Brasil, 2009 a 2011. 
Tabela 3. Risco relativo percentual (\%RR) e intervalos de confiança (IC) dos efeitos sinérgicos da estação do ano, temperatura máxima e umidade para internação e mortalidade por doenças cardiovasculares relacionadas a incrementos de $10 \mu \mathrm{g} / \mathrm{m}^{3}$ de $\mathrm{PM}_{2,5}$ por defasagem simples. Cuiabá e Várzea Grande, MT, Brasil, 2009 a 2011.

\begin{tabular}{|c|c|c|c|c|c|}
\hline \multirow{2}{*}{ Variável } & & \multicolumn{2}{|c|}{ Internações } & \multicolumn{2}{|c|}{ Óbitos } \\
\hline & & $\%$ RR & IC95\% & $\%$ RR & IC95\% \\
\hline \multirow{2}{*}{ Estação do ano } & Seca & $2,35^{*}$ & $0,59-4,15$ & $3,43^{*}$ & $0,58-6,35$ \\
\hline & Chuva & 1,05 & $-5,37-7,91$ & $-4,53$ & $-11,95-3,50$ \\
\hline \multirow{2}{*}{ Temperatura máxima } & $>37,9^{\circ} \mathrm{C}$ & 0,95 & $-1,85-3,82$ & $4,90^{*}$ & $0,61-9,38$ \\
\hline & $<37,9^{\circ} \mathrm{C}$ & $-1,80$ & $-4,95-1,45$ & 1,55 & $-3,49-6,86$ \\
\hline \multirow{2}{*}{ Umidade } & $>54,5 \%$ & 0,57 & $-2,75-4,02$ & 2,05 & $-3,68-8,14$ \\
\hline & $<54,5 \%$ & $2,71^{*}$ & $-0,39-5,92$ & $5,35^{*}$ & $-0,20-11,22$ \\
\hline
\end{tabular}

$* p \leq 0,05$

Os resultados das análises de efeito modificador mostraram que a temperatura, a umidade e a estação do ano podem apresentar efeitos sinérgicos tanto das internações quanto dos óbitos por DC relacionados ao $\mathrm{PM}_{2,5^{\circ}} \mathrm{O} \% \mathrm{RR}$ da mortalidade por $\mathrm{DC}$ relacionado ao $\mathrm{PM}_{2.5}$ foi 4,90 (IC95\% -0,61-9,38) em dias cuja temperatura máxima esteve acima de 37,9 $9^{\circ} \mathrm{C}$. O \%RR da mortalidade e das internações por DC relacionado ao $\mathrm{PM}_{25}$ foram 5,35 (IC95\% -0,20-11,22) e 2,71 (IC95\% -0,39-5,92), respectivamente, em dias com umidade relativa do ar abaixo de $54,5 \%$. Durante a estação seca, foi observado \%RR 3,43 (IC95\% 0,58-6,35) para a mortalidade e \%RR 2,35 (IC95\% 0,59-4,15) para as internações. Não foi observada modificação de efeito do $\mathrm{PM}_{2,5}$ pela temperatura máxima relacionada às internações (Tabela 3).

\section{DISCUSSÃO}

Os resultados deste estudo reforçam achados da literatura internacional ${ }^{5}$ e brasileira ${ }^{11,20}$ ao apontar a relação entre $\mathrm{PM}_{2,5}$ e o aumento do risco de internação e mortalidade por DC em adultos com 45 anos ou mais de idade. Os efeitos adversos do PM sobre as internações por DC coincidiram com o mesmo dia do aumento do poluente (Lag0) e diminuíram o risco nos dias subsequentes, enquanto os efeitos do PM sobre a mortalidade por DC aumentou após o aumento do poluente, culminando em mortalidade, principalmente, depois do terceiro dia de exposição (lag3). Essa relação corrobora a literatura, indicando que as DC são desfechos mais inespecíficos e com muitas causas associadas 5 . Assim, apesar de ambos os desfechos estarem relacionados a casos mais graves, as internações apresentam caráter agudo, enquanto a mortalidade pode estar associada à complicação dos agravos ${ }^{20}$.

O calor apresentou efeito sinérgico com o $\mathrm{PM}_{2,5}$ sobre a mortalidade por DC neste estudo. Esse sinergismo entre temperatura e a mortalidade por DC ainda é controverso na literatura científica, mas alguns autores observaram que efeitos adversos do material particulado podem ser mais aparentes em estações mais quentes ${ }^{8,23}$ e em temperaturas mais altas ${ }^{10,17}$. Ren e Tong ${ }^{17}$ observaram \%RR de 2,32 (IC95\% -0,26-4,97) para internações por DC e 6,95 (IC95\% 0,95-13,33) para mortalidade por DC relacionada ao $\mathrm{PM}_{10}$ em dias com temperatura acima de $27^{\circ} \mathrm{C}$ em Brisbane, Austrália, entre 1996 e 2001. Meng et al..$^{10}$ observaram cerca de \%RR de 1,57\% (IC95\% 0,69-2,46) para a mortalidade cardiovascular relacionada ao $\mathrm{PM}_{10}$ em dias com temperatura acima de $30^{\circ} \mathrm{C}$ (> percentil 95) para oito cidades da China entre 2001 e 2008, cujo \%RR foi de 3,19\% (IC95\% 2,55-3,84) para a cidade de Wuhan. Nos Estados Unidos, foi observado, em um estudo ecológico realizado para 207 cidades, aumento da mortalidade por DC relacionada ao $\mathrm{PM}_{2,5}$ nas cidades com maiores temperaturas médias 8 .

A modificação dos efeitos do $\mathrm{PM}_{2,5}$ por altas temperaturas na saúde humana pode estar relacionada à resposta direta ou indireta do organismo ao estresse proveniente do calor ${ }^{18}$. A termorregulação corporal está diretamente ligada à regulação circulatória de um indivíduo. Dessa forma, dias quentes podem sobrecarregar o sistema corpóreo de regulação da temperatura e alterar a resposta fisiológica aos agentes tóxicos, aumentando a vulnerabilidade individual 
aos efeitos do $\mathrm{PM}^{4,17}$. Em dias mais quentes também pode ocorrer uma maior exposição à poluição atmosférica devido à propensão das pessoas a manterem as janelas abertas ou a passar mais tempo ao ar livre ${ }^{23}$. Alguns estudos sugerem que a alta temperatura também pode ser considerada uma medida indireta da composição das partículas atmosféricas urbanas $^{8}$, uma vez que associam-se fortemente ao carbono e sulfato orgânico e elementar ${ }^{24}$, ao ozônio ${ }^{3}$ e à concentração das partículas semi-voláteis ${ }^{21}$.

Nossos resultados apresentaram evidências de que a ação do $\mathrm{PM}_{2.5}$ sobre as internações e a mortalidade por DC pode ser exacerbada em dias de baixa umidade relativa do ar (abaixo de $54,5 \%)$. Os efeitos da umidade relacionados à prevalência de hospitalizações e à mortalidade, assim como seu impacto sobre os efeitos da poluição, são pouco relatados na literatura. Qiu et al. ${ }^{14}$, na China, observaram aumento de 1,67 (IC95\% 1,26-2,08) nas admissões de emergência por doenças isquêmicas do coração relacionadas ao $\mathrm{PM}_{10} \mathrm{em}$ dias em que a umidade relativa do ar encontrava-se abaixo de $80 \%$. Ravljen et al. ${ }^{16}$, na Eslovênia, identificaram que o aumento de $1 \%$ na umidade média diária diminui em cerca de $3 \%$ (IC95\% -6,00--1,00) a incidência diária de síndrome coronariana aguda. Em Cuiabá e Várzea Grande, Rodrigues et al. ${ }^{19}$ observaram que clusters temporais de alto risco para mortalidade por DC apresentaram maiores médias diárias de $\mathrm{PM}_{2,5}$ e menores médias diárias na umidade relativa do ar. Entretanto, a comparação entre esses resultados é dificultada devido às grandes diferenças metodológicas utilizadas.

A sazonalidade é descrita por vários autores como modificadora dos efeitos do PM associados às internações e à mortalidade por $\mathrm{DC}^{8,23,24}$. Alguns estudos apontam as estações do ano como medidas indiretas da temperatura, como Kioumourtzoglou et al. ${ }^{8}$, nos EUA, e Staffogia et al. ${ }^{23}$, na Itália, que encontraram aumento da mortalidade por DC relacionada ao $\mathrm{PM}_{2,5}$ e ao $\mathrm{PM}_{10}$, respectivamente, no verão. Estudos de especiação da química atmosférica sugerem que pode ocorrer a prevalência de componentes mais tóxicos em concentrações mais elevadas durante diferentes épocas do ano, de acordo com as características climáticas e de emissões de poluentes de cada local, sendo a prevalência de chuva o mecanismo mais eficaz para a deposição dos poluentes ${ }^{15,24,25}$. Os achados deste estudo mostram que a estação seca pode aumentar tanto as internações quanto os óbitos por DC relacionados ao $\mathrm{PM}_{2,5}$, o que é compatível com ambas as hipóteses. Em primeiro lugar, porque a estação seca também apresentou temperaturas mais elevadas que a estação chuvosa em nosso banco de dados. Em segundo lugar, porque o período de seca é, também, o período com maior prevalência de queimadas nos municípios e nas regiões Norte e Centro-Oeste do país.

Deve ser levado em consideração que podem existir outras fontes de poluição do ar, como o tráfego veicular, queima de lixo urbano ou o próprio transporte de poluentes atmosféricos provenientes da queima de biomassa e emissões industriais de cidades vizinhas. Apesar de também influenciarem de maneira importante a morbimortalidade por DC, essas fontes não foram contabilizadas em nossas análises, pois não haviam dados disponíveis sobre estas variáveis. A condição climática local também deve ser considerada. Cuiabá e Várzea Grande estão localizadas em uma depressão com extensos chapadões à sua borda e apresentam grande periodicidade de situações de estabilidade atmosférica (céu limpo e baixa velocidade do vento), o que pode dificultar a dispersão dos poluentes e aumentar a incidência de ilhas de calor e episódios de inversão térmica. Além disso, a estação seca pode caracterizar uma medida indireta dos efeitos da baixa umidade ${ }^{19,22}$, também observados neste estudo.

Como limitações do estudo, podemos destacar o viés de preenchimento inerente ao uso da base de dados de internações e mortalidade do SUS que pode não retratar precisamente a distribuição das variáveis estudadas. No entanto, estudos têm indicado confiabilidade dos dados contidos nos sistemas de informação de saúde $e^{9}$ Outro ponto importante está relacionado à utilização de valores estimados para o PM, obtidos por técnicas de sensoriamento remoto, que podem subestimar ou não condizer com valores reais da exposição pessoal; contudo, seu uso mostra-se factível e vantajoso tanto para este estudo, quanto para regiões remotas que não apresentam estações de monitoramento atmosférico ${ }^{1,2}$. É importante mencionar que o caráter ecológico das nossas análises não permite identificar qualquer efeito causal, ou seja, a associação observada entre os agregados não significa que a mesma associação ocorra em nível individual. 
Concluiu-se que o $\mathrm{PM}_{2,5}$ apresenta associação com internações e a mortalidade por DC em cidades de porte médio. A baixa umidade relativa do ar e a estação seca podem aumentar tanto as internações quanto os óbitos por $\mathrm{DC}$ relacionados ao $\mathrm{PM}_{2,5}$ O calor está relacionado ao aumento do risco de mortalidade por DC associado ao $\mathrm{PM}_{2,5}$.

Este é o primeiro estudo que aborda as interações dos fatores climáticos com a poluição na região do Cerrado. A utilização de dados estimados para a poluição, obtidos por técnicas de sensoriamento remoto, proporciona resultados compatíveis aos encontrados em outros estudos ecológicos, indicando que essa ferramenta é eficaz na obtenção das concentrações de PM. Os resultados desta pesquisa podem ser utilizados para uma avaliação acerca do aprimoramento da qualidade dos dados de mortalidade por DC e de poluição atmosférica, para fundamentar estudos futuros e, principalmente, para auxiliar no planejamento de estratégias voltadas à mitigação do impacto da poluição do ar na saúde humana.

\section{REFERÊNCIAS}

1. Andrade Filho VS, Artaxo P, Hacon S, Carmo CN, Cirino G. Aerosols from biomass burning and respiratory diseases in children, Manaus, Northern Brazil. Rev Saude Publica. 2013;47(2):239-47. https://doi.org/10.1590/S0034-8910.2013047004011

2. Baars H, Ansmann A, Althausen D, Engelmann R, Heese B, Müller D, et al. Aerosol profiling with lidar in the Amazon Basin during the wet and dry season. J Geophys Res Atmospheres. 2012;117(D21):1-16. https://doi.org/10.1029/2012JD018338

3. Bell ML, McDermott A, Zeger SL, Samet JM, Dominici F. Ozone and short-term mortality in 95 US urban communities, 1987-2000. JAMA. 2004;292(19):2372-8. https://doi.org/10.1001/jama.292.19.2372

4. Cheng X, Su H. Effects of climatic temperature stress on cardiovascular diseases. Eur J Intern Med. 2010;21(3):164-7. https://doi.org/10.1016/j.ejim.2010.03.001

5. Franchini M, Mannucci PM. Air pollution and cardiovascular diseases. Thromb Res. 2012;129(3):230-4. https://doi.org/10.1016/j.thromres.2011.10.030

6. Ignotti E, Valente JG, Longo KM, Freitas SR, Hacon SS, Artaxo Netto P. Impact on human health of particulate matter emitted from burnings in the Brazilian Amazon region. Rev Saude Publica. 2010;44(1):121-30. https://doi.org/10.1590/S0034-89102010000100013

7. Junger W, Leon AP. Ares: a library for time series analysis in air pollution and health effects studies using R. Epidemiology. 2009;20(6):S217. https://doi.org/10.1097/01.ede.0000362727.55583.4a

8. Kioumourtzoglou MA, Schwartz J, James P, Dominici F, Zanobetti A. PM 2.5 and mortality in 207 US cities: modification by temperature and city characteristics. Epidemiology. 2016;27(2):221-7. https://doi.org/10.1097/EDE.0000000000000422

9. Lemos C, Chaves LDP, Azevedo ALCS. Sistemas de informação hospitalar no âmbito do SUS: revisão integrativa de pesquisas. Rev Eletron Enferm [Internet]. 2010 [citado $17 \mathrm{dez}$ 2015];12(1):177-85. Disponível em: http://www.fen.ufg.br/revista/v12/n1/v12n1a22.htm

10. Meng X, Zhang Y, Zhao Z, Duan X, Xu X, Kan H. Temperature modifies the acute effect of particulate air pollution on mortality in eight Chinese cities. Sci Total Environ. 2012;435-436:215-21. https://doi.org/10.1016/j.scitotenv.2012.07.008

11. Nardocci AC, Freitas CU, Ponce de Leon ACM, Monteiro AC, Junger WL, Gouveia NC. Poluição do ar e doenças respiratórias e cardiovasculares: estudo de séries temporais em Cubatão, São Paulo, Brasil. Cad Saude Publica 2013;29(9):1867-76. https://doi.org/10.1590/0102-311X00150012

12. Nunes KVR, Ignotti E, Hacon SS. Circulatory disease mortality rates in the elderly and exposure to $\mathrm{PM}_{2.5}$ generated by biomass burning in the Brazilian Amazon in 2005. Cad Saude Publica. 2013;29(3):589-98. https://doi.org/10.1590/S0102-311X2013000300016

13. Pinheiro SLLA, Saldiva PHN, Schwartz J, Zanobetti A. Isolated and synergistic effects of PM ${ }_{10}$ and average temperature on cardiovascular and respiratory mortality. Rev Saude Publica. 2014;48(6):881-8. https://doi.org/10.1590/S0034-8910.2014048005218

14. Qiu H, Yu IT, Wang X, Tian L, Tse LA, Wong TW. Cool and dry weather enhances the effects of air pollution on emergency IHD hospital admissions. Int / Cardiol. 2013;168(1):500-5. https://doi.org/10.1016/j.ijcard.2012.09.199 
15. Qiu H, Yu IT, Wang X, Tian L, Tse LA, Wong TW. Differential effects of fine and coarse particles on daily emergency cardiovascular hospitalizations in Hong Kong. Atmos Environ. 2013;64:296-302. https://doi.org/10.1016/j.atmosenv.2012.09.060

16. Ravljen M, Bilban M, Kajfež-Bogataj L, Hovelja T, Vavpoti D. Influence of daily individual meteorological parameters on the incidence of acute coronary syndrome. Int J Environ Res Public Health. 2014;11(11):11616-26. https://doi.org/10.3390/ijerph111111616

17. Ren C, Tong S. Temperature modifies the health effects of particulate matter in Brisbane, Australia. Int J Biometeorol. 2006;51(2):87-96. https://doi.org/10.1007/s00484-006-0054-7

18. Roberts S. Interactions between particulate air pollution and temperature in air pollution mortality time series studies. Environ Res. 2004;96(3):328-37. https://doi.org/10.1016/j.envres.2004.01.015

19. Rodrigues PCO, Santos ES, Ignotti E, Hacon SS. Space-time analysis to identify areas at risk of mortality from cardiovascular disease. BioMed Res Int. 2015;2015:ID 841645. https://doi.org/10.1155/2015/841645

20. Romieu I, Gouveia N, Cifuentes LA, Leon AP, Junger W, Vera J, et al. Multicity study of air pollution and mortality in Latin America (the ESCALA study). Res Rep Health Eff Inst. 2012;(171):5-86

21. Seinfeld JH, Pandis SN. Atmospheric chemistry and physics: from air pollution to climate change. Hoboken (NJ): Wiley; 2006.

22. Silva AMC, Mattos IE, Freitas SR, Longo KM, Hacon SS. Material particulado $\left(\mathrm{PM}_{2,5}\right)$ de queima de biomassa e doenças respiratórias no sul da Amazônia brasileira. Rev Bras Epidemiol . 2010;13(2):337-51. https://doi.org/10.1590/S1415-790X2010000200015

23. Stafoggia M, Schwartz J, Forastiere F, Perucci CA; SISTI Group. Does temperature modify the association between air pollution and mortality? A multicity case-crossover analysis in Italy. Am J Epidemiol. 2008;167(12):1476-85. https://doi.org/10.1093/aje/kwn074

24. Tai APK, Mickley LJ, Jacob DJ. Correlations between fine particulate matter $\left(\mathrm{PM}_{2.5}\right)$ and meteorological variables in the United States: implications for the sensitivity of $\mathrm{PM}_{25}$ to climate change. Atmos Environ. 2010;44(32):3976-84. https://doi.org/10.1016/j.atmosenv.2010.06.060

25. Villalobos AM, Barraza F, Jorquera $H$, Schauer JJ. Chemical speciation and source apportionment of fine particulate matter in Santiago, Chile, 2013. Sci Total Environ. 2015;512-513:133-42. https://doi.org/10.1016/j.scitotenv.2015.01.006

Financiamento: Rede Brasileira de Pesquisas sobre Mudanças Climáticas Globais (convênio FINEP/Rede CLIMA 01.08.0405.01).

Contribuição dos Autores: PCOR realizou concepção e desenho do estudo, aquisição de dados, análise e interpretação dos dados e elaboração do manuscrito. SLP participou da analise e interpretação dos resultados. WJ, EI e SSH participaram da interpretação dos dados e revisão crítica do manuscrito. Todos os autores aprovaram a versão final do manuscrito e assumem a responsabilidade pública pelo conteúdo do artigo.

Conflito de Interesses: Os autores declaram não haver conflito de interesses. 